\title{
PRIMER REGISTRO DE Dusicyon gymnocercus (FISHER, 1814) (CARNIVORA: CANIDAE) EN EL PLEISTOCENO DE ENTRE RÍOS (ARGENTINA)
}

\author{
Francisco Juan PREVOSTI ${ }^{1}$, Jorge Ignacio NO- \\ RIEGA ${ }^{2}$, César Mariano GARCÍA ESPONDA ${ }^{3}$ y \\ Brenda Soledad FERRERO ${ }^{2}$
}

\begin{abstract}
${ }^{1}$ Departamento Paleontología Vertebrados, Museo de La Plata, Paseo del Bosque s/n, 1900 La Plata, Buenos Aires, Argentina. CONICET. protocyon@hotmail.com ${ }^{2}$ Laboratorio de Paleontología de Vertebrados, CICyTTP-CONICET, Matteri y España, 3105 Diamante, Argentina, brendaferrero@yahoo.com.ar, cidnoriega@infoaire.com.ar

${ }^{3}$ Cátedra de Anatomía Comparada, Facultad de Ciencias Naturales y Museo (UNLP), calle 64 n³ 3, 1900, La Plata, Argentina, cesponda@fcnym.unlp.edu.ar
\end{abstract}

Prevosti, F. J., Noriega, J. I., García Esponda, C. M. \& Ferrero, B. S. 2005. Primer registro de Dusicyon gymnocercus (Fisher, 1814) (Carnivora: Canidae) en el Pleistoceno de Entre Ríos (Argentina). [First record of Dusicyon gymnocercus (Fischer, 1814) (Carnivora: Canidae) from the Pleistocene of Entre Ríos (Argentina).] Revista Española de Paleontología, 20 (2), 159-167. ISSN 0213-6937.

\begin{abstract}
The first fossil record of Dusicyon gymnocercus (Fischer, 1814) from Entre Ríos province in Argentina is described from the Arroyo Feliciano Formation (Lujanian; Late Pleistocene-Early Holocene). The specimen (CICYTTP-PV-M-22) is a nearly complete skull without mandibles. The morphology and size of the dentition and skull is indistinguishable from the recent species Dusicyon gymnocercus. The multivariate analysis performed agrees with this assignation. This skull is the first fossil record of a canid in Entre Ríos province and the Argentinian Mesopotamia, and expands the geographic distribution of Dusicyon gymnocercus during the Lujanian.
\end{abstract}

Keywords: Canidae, Dusicyon gymnocercus, Arroyo Feliciano Formation, Late Pleistocene-early Holocene, Argentina.

\section{RESUMEN}

Se reporta el primer registro paleontológico de Dusicyon gymnocercus (Fischer, 1814) procedente de la Formación Arroyo Feliciano (Edad Lujanense; Pleistoceno Tardío-Holoceno Temprano) de la provincia de Entre Ríos, Argentina. El ejemplar (CICYTTP-PV-M-22) consiste en un cráneo completo sin mandíbula cuya morfología cráneo-dentaria es indistinguible de la especie viviente Dusicyon gymnocercus. El tamaño general del cráneo y las medidas cráneo-dentarias tomadas se encuentran dentro del rango de variación de Dusicyon gymnocercus. Los análisis multivariados permitieron llegar a la misma conclusión. Este constituye el primer registro de un cánido para el Lujanense de Entre Ríos y la Mesopotamia Argentina y amplía la distribución geográfica de Dusicyon gymnocercus durante el Lujanense.

Palabras claves: Canidae, Dusicyon gymnocercus, Formación Arroyo Feliciano, Pleistoceno tardíoHoloceno temprano, Argentina.

\section{INTRODUCCIÓN}

Dusicyon gymnocercus (Fischer, 1814), conocida vulgarmente como zorro pampa, es un especie relativamente común, que habita actualmente los "pastizales" y otros ambientes abiertos de gran parte de Argentina, Uruguay, Paraguay, el sur de Brasil y el este de Bolivia (Cabrera, 1957; Ginsberg \& Macdonald, 1990; Macdonald \& Sillero-Zubiri, 2004). En la República Argentina se distribuye desde el río Negro, hasta Misiones, Formosa, Jujuy y 
Salta (Cabrera, 1957; Jayat et al., 1999). Es una especie oportunista, que posee una dieta omnívora compuesta principalmente por pequeños y medianos mamíferos, insectos y frutos (Crespo, 1971). Los restos fósiles más antiguos de D. gymnocercus corresponden al Pleistoceno Temprano-Medio (Ensenadense) de la región Pampeana (Buenos Aires, Argentina) y Tarija (Tarija, Bolivia) (Kraglievich, 1930; Berman, 1994). Durante el Pleistoceno Tardío (Lujanense) se lo registra en la provincia de Buenos Aires, Minas Gerais (Brasil) y Uruguay (Winge, 1895; Kraglievich, 1930; Ubilla \& Perea, 1999).

El estatus genérico de los zorros sudamericanos fue revisado por numerosos autores (e.g. Kraglievich, 1930; Cabrera, 1931; Osgood, 1934; Langguth, 1975; Clutton-Brock et al., 1976; Van Gelder, 1978; Berta, 1987; Zunino et al., 1995) quienes utilizaron diferentes nombres genéricos (e.g. Pseudalopex Burmeister, 1856; Dusicyon Hamilton Smith, 1839; Lycalopex Burmeister, 1854) para agrupar a las especies de zorros de campo [i.e. D. sechurae (Thomas, 1900); D. culpaeus (Molina, 1792); D. gymnocercus; D. australis (Kerr, 1792); D. griseus (Gray, 1837); D. vetulus (Lund, 1842), y D. fulvipes (Martin, 1837)]. Según un análisis filogenético llevado a cabo por Tedford et al. (1995), sobre la base de caracteres osteológicos y dentarios, este grupo es parafilético, motivo por el cual los autores restringen Dusicyon para D. australis, Lycalopex para L. vetulus, Pseudalopex para $P$. culpaeus, quedando el resto en "Pseudalopex". Recientemente estos datos fueron combinados con otra evidencia morfológica, más caracteres de comportamiento, historia de vida y moleculares (Zrzavý \& Řičánková, 2004). En este último estudio las especies mencionadas constituyen un grupo monofilético, por lo que siguiendo los principios de la clasificación cladística deben ser incluidas en un mismo taxón, siendo Dusicyon el nombre que corresponde por prioridad.
Por otra parte, Zunino et al. (1995) consideran que $D$. griseus y D. gymnocercus son sinónimos. Sin embargo, esta hipótesis no fue debidamente contrastada ni seguida por autores posteriores (Wayne et al., 1997; Wang et al. 2004; Macdonald \& Sillero-Zubiri, 2004), para lo cual sería necesario aumentar el número de ejemplares y localidades, así también como la utilización de técnicas moleculares (ADN). La sistemática de los zorros fósiles todavía no ha superado la etapa tipológica, ya que no tiene en cuenta la variación (geográfica, ontogenética, sexual) existente en las especies vivientes. Esto queda reflejado en los caracteres utilizados para diagnosticarlas (e.g. Berman, 1994; vide infra).

En este trabajo se describe un nuevo cráneo de un zorro del Pleistoceno Tardío, discutiendo su asignación taxonómica. Se realizaron estudios morfológicos descriptivos, métricos y estadísticos de las tres especies que actualmente viven en la Argentina (D. culpaeus, D. griseus, D. gymnocercus), con el objetivo de identificar con mayor confianza taxonómica el material fósil, teniendo en cuenta la variación observada en las especies vivientes. Estos análisis permitieron asignar el fósil a $D$. gymnocercus, que constituye el primer registro de un cánido de antigüedad Pleistocena para la provincia de Entre Ríos en Argentina.

\section{MATERIALES Y MÉTODOS}

El ejemplar fósil fue comparado con 62 ejemplares de $D$. gymnocercus, 25 de D. griseus y 22 de D. culpaeus (véase Apéndice). Sobre este material se tomaron 17 medidas craneanas, en milímetros, utilizando calibre digital, con una precisión de 0,01 mm, según se indica en la Tabla 1 .

Se realizó un análisis de componentes principales a partir de la matriz de varianza-covarianza utilizando el programa Statistica

\section{Abreviatura Descripción}

\begin{tabular}{|c|c|}
\hline LCB & Longitud condilobasal, desde el margen anterior del foramen magno hasta la parte más anterior de los premaxilares, tomada en el plano sagital. \\
\hline $\mathrm{LCr}$ & Longitud del cráneo, desde el extremo anterior de los premaxilares hasta el punto de intersección de las suturas sagital y lambdoidea. \\
\hline LP & Longitud palatal, desde el extremo anterior de los premaxilares hasta el margen posterior del paladar. \\
\hline LR & Longitud del rostro, desde el extremo anterior de los premaxilares hasta el borde de la órbita al nivel de la sutura lacrimo-yugal. \\
\hline LB & Longitud de la bulla, entre el foramen rasgado medio y la sutura bulla-apófisis paraoccipital. \\
\hline AIO & Ancho interorbitario mínimo, entre los frontales a nivel de las órbitas, por delante de los procesos postorbitarias. \\
\hline $\mathrm{CPO}$ & Ancho mínimo de la constricción postorbitaria. \\
\hline APPO & Ancho máximo entre procesos postorbitarios. \\
\hline AR & Ancho del rostro entre los maxilares a nivel de los alvéolos de los caninos. \\
\hline AM & Ancho del paladar entre el borde labial de los alvéolos de los M1. \\
\hline $\mathrm{ACO}$ & Ancho máximo entre los cóndilos occipitales. \\
\hline SDS & Largo de la serie dentaria superior, entre el borde anterior del $\mathrm{C}$ y el margen posterior del M2 a nivel alveolar. \\
\hline LC & Longitud anteroposterior del canino superior a nivel alveolar. \\
\hline LP4 & Longitud anteroposterior del P4 a nivel alveolar. \\
\hline AP4 & Ancho del P4 a nivel alveolar. \\
\hline LM1 & Longitud anteroposterior del M1 a nivel alveolar M1. \\
\hline AM1 & Áncho máximo del M1 a nivel alveolar. \\
\hline
\end{tabular}

Tabla 1. Medidas cráneo-dentarias utilizadas en este estudio.

Skull and tooth measurements used in this study. 


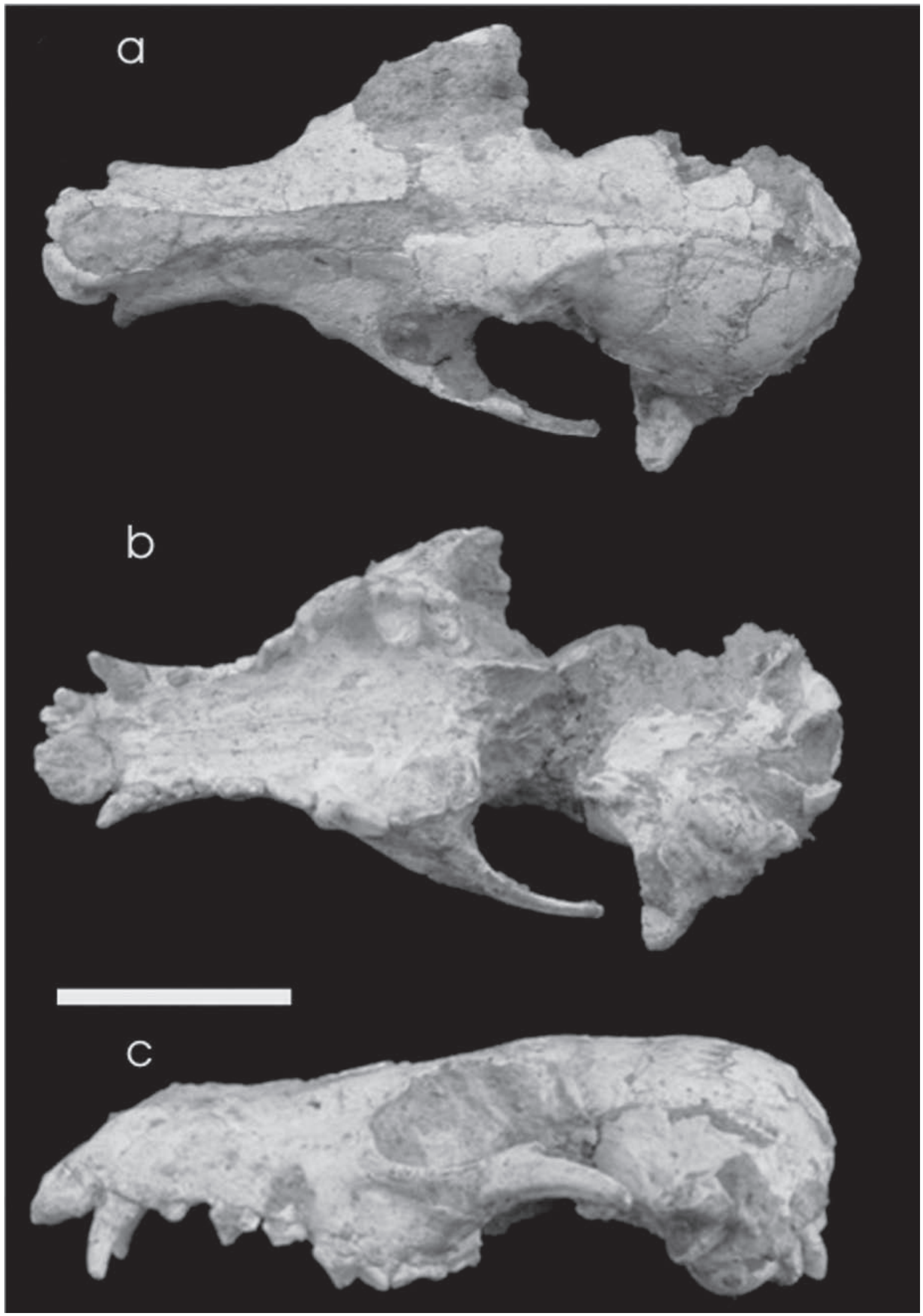

Figura 1. Cráneo de Dusicyon gymnocercus (Fisher, 1814) (CICYTTP-PV-M-22) encontrado en el Pleistoceno Tardío del Arroyo Ensenada (Entre Ríos, Argentina). a: vista dorsal; b: vista ventral; c: vista lateral. Escala: $5 \mathrm{~cm}$.

Skull of Dusicyon gymnocercus (Fisher, 1814) (CICYTTP-PV-M-22) founded in Late Pleistocene from Arroyo Ensenada (Entre Ríos, Argentina). a: dorsal view; b: ventral view; : lateral view. Escale: $5 \mathrm{~cm}$. 
6.0 (Statsoft inc., 2001). Los datos fueron transformados a sus logaritmos naturales para homogeneizar sus varianzas. Con este programa también se hizo un análisis discriminante (AD) y un análisis canónico de la varianza (ACV). En el AD se le otorgo la misma probabilidad a todos los grupos, constituidos por las tres especies vivientes.

En los aspectos cronoestratigráficos y bioestratigráficos se siguió a Cione \& Tonni (1999, 2001).

Abreviaturas: CICYTTP-PV-M: Laboratorio de Paleontología de Vertebrados del Centro de Investigaciones Científicas y Transferencia de Tecnología a la Producción de Diamante; MLP Departamento Científico de Vertebrados del Museo de La Plata, Sección Mastozoología; MACN-Ma: Colección Nacional de Mastozoología del Museo Argentino de Ciencias Naturales Bernardino Rivadavia; I: incisivo superior; $C$ : canino superior; $\mathrm{P}$ : premolar superior; M: molar superior.

\section{SISTEMÁTICA PALEONTOLÓGICA}

ORDEN CARNIVORA Bowdich, 1821 Familia Canidae Fischer, 1817

Género Dusicyon Hamilton Smith, 1839

Especie tipo: Canis antarticus Bechstein, 1799

\section{Dusicyon gymnocercus (Fischer, 1814)}

Fig. 1, Tabla 2

Material asignado: CICYTTP-PV-M-22: cráneo casi completo sin mandíbula (Fig. 1).

Procedencia geográfica y estratigráfica: El material proviene de las barrancas del arroyo Ensenada en el Departamento de Diamante de la provincia de Entre Ríos, Argentina (Fig. 2). El mismo fue colectado en sedimentos limosos loessoides, marrones claros, con abundantes intercalaciones lenticulares de conglomerados formados por concreciones carbonáticas. Esta unidad corresponde a la Formación Arroyo Feliciano y constituye la terraza más alta de los principales colectores de la provincia y de los tramos inferiores de sus afluentes (Iriondo et al., 1985; Iriondo, 1996). La antiguiedad asignada a la misma sobre la base de la composición de su paleomastofauna corresponde a la base del Lujanense (Pleistoceno Tardío; Noriega et al., 2004).

Descripción: El cráneo se encuentra casi completo, faltando gran parte del arco cigomático derecho (Fig. 1). El proceso cigomático del escamoso y el extremo caudal del yugal se encuentran rotos

\begin{tabular}{|c|c|c|c|c|}
\hline & CICYTTP-PV-M-22 & $\begin{array}{c}\text { D. griseus } \\
\mathrm{X}+\text { /- DS (N) }\end{array}$ & $\begin{array}{c}\text { D. culpaeus } \\
\mathrm{X}+\text { /- DS (N) }\end{array}$ & $\begin{array}{c}\text { D. gymnocercus } \\
\mathrm{X}+/ \text { - DS (N) }\end{array}$ \\
\hline LCB & 140,9 & $121.70+/-5.59(25)$ & $166.05+/-7.94(21)$ & $137.15+/-6.86(30)$ \\
\hline LCR & 143,7 & $125.48+/-6.20(25)$ & $174.87+/-7.88(22)$ & $141.97+/-7.31(30)$ \\
\hline LP & 73,88 & $64.89+/-3.54(25)$ & $88.14+/-5.03(22)$ & $71.41+/-3.66(30)$ \\
\hline LR & 61,4 & $52.60+/-3.09(25)$ & $73.82+/-4.01(22)$ & $59.6+/-3.49(30)$ \\
\hline LB & 20,5 & $19.31+/-1.05(25)$ & $22.99+/-1.15(21)$ & $21.45+/-1.05(30)$ \\
\hline AIO & 27,1 & $21.66+/-1.63(25)$ & $29.38+/-2.14(22)$ & $25.56+/-1.83(30)$ \\
\hline CPO & 23,7 & $23.88+/-1.29(24)$ & $26.91+/-1.68(22)$ & $24.65+/-2.05(30)$ \\
\hline APPO & 33,9 & $29.60+/-2.15(23)$ & $37.32+/-2.80(22)$ & $35.08+/-2.96(30)$ \\
\hline AR & 26,4 & $18.46+/-1.07(25)$ & $28.06+/-1.44(22)$ & $22.25+/-1.43(30)$ \\
\hline AM & 43,4 & $35.43+/-1.63(25)$ & $47.72+/-2.17(22)$ & $38.81+/-1.79(30)$ \\
\hline ACO & 26,6 & $23.38+/-1.48(24)$ & $29.88+/-0.98(21)$ & $27.66+/-3.27(30)$ \\
\hline SDS & 65,1 & $56.67+/-2.63(25)$ & $73.49+/-3.13(22)$ & $60.94+/-2.97(30)$ \\
\hline LC & 7,2 & $5.52+/-0.68(25)$ & $10.00+/-3.13(22)$ & $6.42+/-0.65(30)$ \\
\hline LP4 & 14,5 & $11.36+/-0.57(25)$ & $15.62+/-0.97(22)$ & $12.18+/-0.81(30)$ \\
\hline AP4 & 6,8 & $5.36+/-0.44(25)$ & $7.22+/-0.57(22)$ & $5.91+/-0.54(30)$ \\
\hline LM1 & 9,3 & $7.70+/-0.84(25)$ & $8.57+/-0.74(22)$ & $8.12+/-0.63(30)$ \\
\hline AM1 & 14,4 & $11.65+/-0.59(25)$ & $13.85+/-0.62(22)$ & $12.05+/-0.87(29)$ \\
\hline
\end{tabular}

Tabla 2. Medidas cráneo-dentarias (mm.) del ejemplar CICyTTP-PV-M-22 y de las especies vivientes Dusicyon culpaeus (Molina, 1782), Dusicyon griseus (Gray, 1837) y Dusicyon gymnocercus (Fisher, 1814). X: media; DS: desvío estándar; N: número de ejemplares medidos.

Craneodentary measures $(\mathrm{mm})$ of the CICYTTP-PV-M-22 and the recent species Dusicyon culpaeus (Molina, 1782), Dusicyon griseus (Gray, 1837) and Dusicyon gymnocercus (Fisher, 1814). X: mean; DS: Standard deviation; N: simple size. 


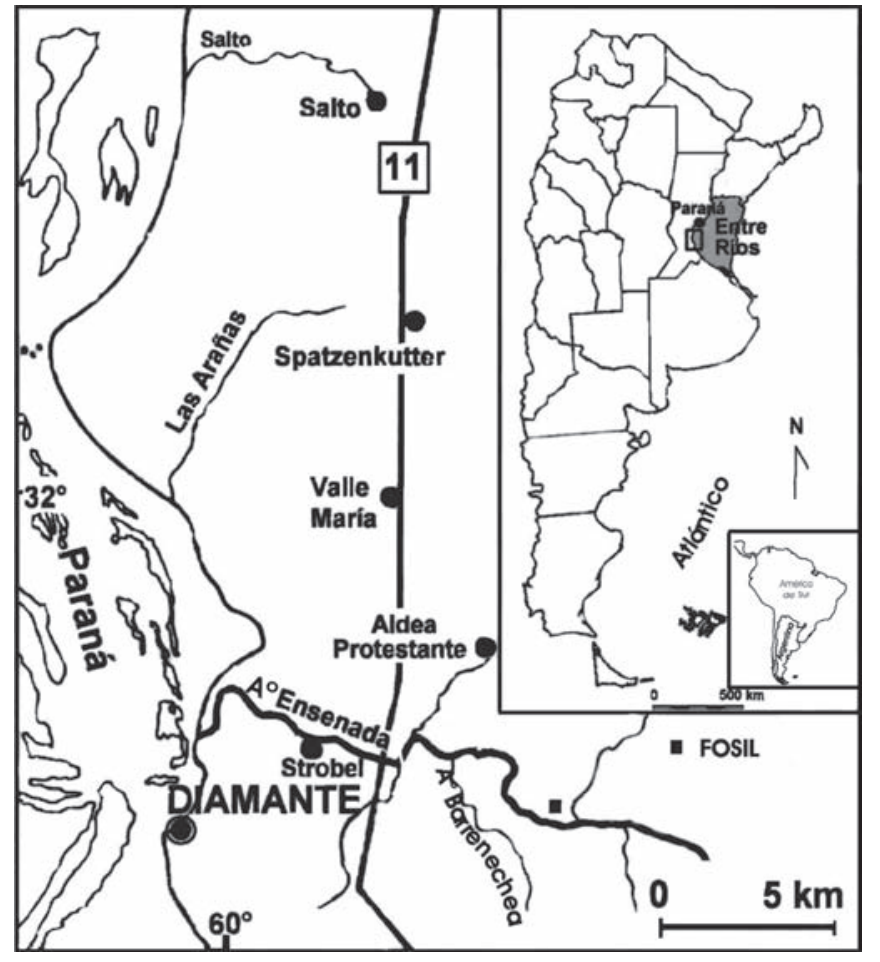

Figura 2. Mapa de la República Argentina mostrando la ubicación geográfica del yacimiento paleontológico Arroyo Ensenada.

Location map of the Arroyo Ensenada paleontological site.

en el arco cigomático izquierdo. También está ausente una porción del lado derecho de la caja craneana que incluye a la bulla timpánica. Las crestas temporales y lambdoidea se encuentran rotas. Este ejemplar ha sido comprimido y fracturado, posiblemente durante la diagénesis fósil. El rostro es proporcionalmente corto con relación a la caja craneana y ancho a la altura de los caninos. El borde caudal del paladar se extiende hasta la mitad de los M2. Los procesos postorbitarios del frontal son pequeños y puntiagudos. La constricción postorbitaria se halla inmediatamente por detrás de los procesos postorbitales y es más angosta que el ancho interorbitario mínimo.

Las crestas temporales forman una amplia área liriforme y sólo cerca del inion se unen para formar una cresta sagital. La sutura entre el basiesfenoides y el basioccipital se encuentra totalmente obliterada.

La dentición está bien preservada, a excepción de los P1, P2 derecho e I1 derecho, que se hallan rotos. Presenta un marcado desgaste dentario en los I2-3 derechos, los caninos y las cúspides principales de los P4-M2. Una concreción de carbonato de calcio cubre a los I1-3 izquierdos. El I3 presenta una cúspide principal y carece de cúspides accesorias. Los P2-3 son birradiculados y presentan una cúspide principal, pero sólo en el P3 se observa una pequeña cúspide cingular distal. El P4 presenta un protocono grande que se dirige mesiolingualmente y sobrepasa el borde mesial del paracono. El cíngulo lingual está bien marcado. Los M1-2 son proporcionalmente grandes con relación al P4. En estos molares el protocono, el hipocono y el cíngulo labial están bien desarrollados. En el M1 hay un gran metacónulo que falta en el M2.

\section{DISCUSIÓN Y CONCLUSIONES}

La obliteración completa de la sutura basioccipital-basiesfenoides y parcial de la sutura premaxilar-maxilar, así como el grado de desgaste dentario, indican que el ejemplar fósil es un adulto (Crespo, 1971).

La morfología de este cráneo presenta los caracteres que clásicamente se usaron para reconocer a $D$. gymnocercus (e.g. tamaño; desarrollo de cresta sagital; forma de la constricción postorbitaria; forma de los molares superiores; índice molar [LM1+LM2)/LP4]) en comparación con $D$. culpaeus y D. griseus. (Kraglievich, 1930; Osgood, 1934). Los valores de las medidas del ejemplar CICYTTP-PVM-22 son similares a aquellos observados por Zunino et al. (1995: Tabla 2, Apéndice) en poblaciones de D. gymnocercus (sensu stricto) de las provincias de La Pampa y Buenos Aires.

El primer componente del análisis de componentes principales explica el 84,85\% de la variación total, mientras que el segundo componente sólo el 4,55 \% (Tabla 3). En el gráfico de dispersión de los dos primeros componentes (Fig. 3a), las especies vivientes se distribuyen a lo

\begin{tabular}{|l|c|c|}
\hline & 98145,451 & 98145,451 \\
\hline 98145,451 & $-0,247$ & 0,033 \\
\hline LCr & $-0,261$ & 0,041 \\
\hline LP & $-0,247$ & 0,013 \\
\hline LR & $-0,269$ & 0,059 \\
\hline LB & $-0,136$ & 0,170 \\
\hline AIO & $-0,237$ & 0,403 \\
\hline CPO & $-0,092$ & 0,340 \\
\hline APPO & $-0,176$ & 0,558 \\
\hline AR & $-0,317$ & 0,124 \\
\hline AM & $-0,235$ & $-0,031$ \\
\hline ACO & $-0,199$ & 0,171 \\
\hline SDS & $-0,213$ & $-0,008$ \\
\hline LC & $-0,475$ & $-0,448$ \\
\hline LP4 & $-0,254$ & $-0,206$ \\
\hline AP4 & $-0,243$ & $-0,191$ \\
\hline LM1 & $-0,105$ & $-0,183$ \\
\hline AM1 & $-0,150$ & $-0,128$ \\
\hline Autovalor & 0,277 & 0,015 \\
\hline
\end{tabular}

Tabla 3. Primeros dos autovectores y autovalores del análisis de componentes principales.

Two first eigenvectors and eigenvalues of the principal component analysis. 

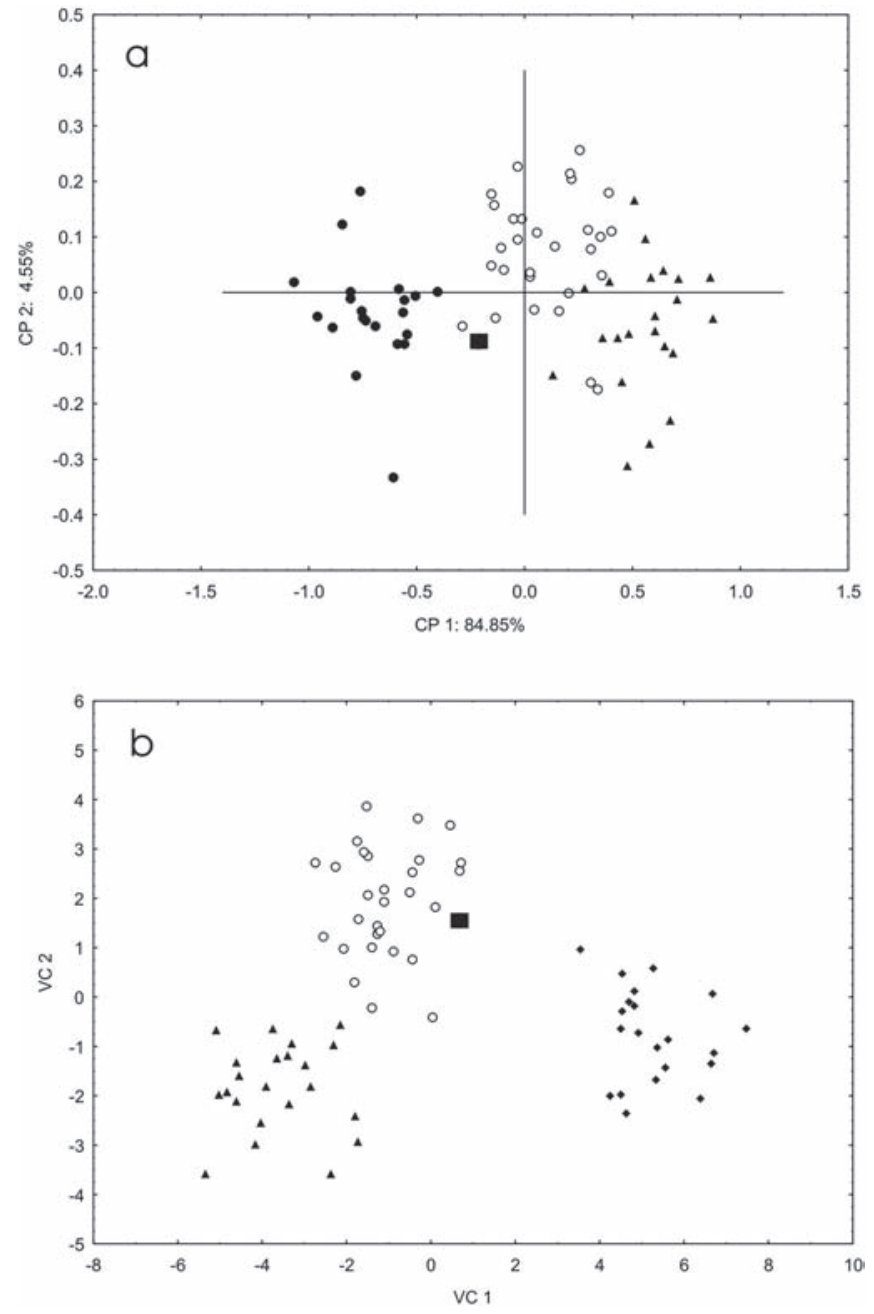

Figura 3. a: gráfico de dispersión de los primeros dos componentes principales $(\mathrm{CP})$ del ACP. b: gráfico de dispersión de las dos variables canónicas (VC) del ACV. Triángulos negros: Dusicyon griseus (Gray, 1837); círculos vacíos: Dusicyon gymnocercus (Fisher, 1814); círculos negros: Dusicyon culpaeus (Molina, 1782); cuadrado: CICYTTP-PV-M-22.

a: biplot of the two first principal components $(C P)$ of the ACP. $\boldsymbol{b}$ : biplot of the canonical variables $(V C)$ of the ACV. Black triangles: Dusicyon griseus (Gray, 1837); empty circles: Dusicyon gymnocercus (Fisher, 1814); black circles: Dusicyon culpaeus (Molina, 1782); square: CICYTTP-PV-M-22.

largo del componente 1 en función de su tamaño, a pesar de que se observa una superposición parcial entre D. griseus y D. gymnocercus. Este componente es interpretado como un vector que refleja el tamaño, ya que todas las variables contribuyen en el mismo sentido y con un peso relativamente similar. A lo largo del primer componente se ubica $D$. griseus (la especie más pequeña) a la derecha y D. culpaeus (la especie de mayor tamaño) a la izquierda, quedando $D$. gymnocercus entre ambas. El componente 2 no permite separar a las especies vivientes ya que las mis-

\begin{tabular}{|l|c|c|}
\hline & VC 1 & VC 2 \\
\hline LCB & 0,339 & $-17,718$ \\
\hline LCr & 34,026 & $-2,811$ \\
\hline LP & 1,486 & 15,847 \\
\hline LR & $-15,048$ & $-16,595$ \\
\hline LB & $-4,881$ & $-3,218$ \\
\hline AIO & $-0,899$ & 0,616 \\
\hline CPO & 4,470 & 4,445 \\
\hline APPO & $-2,704$ & $-10,661$ \\
\hline AR & 5,716 & $-10,069$ \\
\hline AM & 3,400 & 1,838 \\
\hline ACO & 1,504 & $-0,220$ \\
\hline SDS & $-5,414$ & 33,464 \\
\hline LC & 1,904 & 4,778 \\
\hline LP4 & 2,240 & 5,160 \\
\hline AP4 & 2,302 & $-2,661$ \\
\hline LM1 & $-2,895$ & $-1,630$ \\
\hline AM1 & $-1,882$ & $-0,681$ \\
\hline Coeficiente & $-117,669$ & 7,146 \\
\hline
\end{tabular}

Tabla 4. Coeficientes no estandarizados de las dos primeras variables canónicas (VC).

Raw coefficients of the first two canonical variables (VC).

mas se superponen ampliamente. El CICYTTP-PV-M-22 se encuentra dentro del rango de dispersión de D. gymnocercus. La primera variable canónica permite separar a $D$. culpaeus y parcialmente a $D$. griseus de $D$. gymnocercus, aunque la segunda los separa claramente (Fig. 3b; Tabla 4). El análisis discriminante reclasificó correctamente a todos ejemplares vivientes y determinó al CICYTTP-PVM-22 como D. gymnocercus con una probabilidad posterior de 0,999. En resumen, los resultados del análisis de componentes principales y del análisis discriminante sustentan la identificación taxonómica basada en los caracteres clásicamente usados para distinguir a las tres especies vivientes.

Berman (1994) fue el último revisor de las especies de zorros fósiles de la región pampeana. Este autor reconoce tres especies extintas para el Plioceno Tardío-Holoceno de la región: D. avus (Burmeister, 1866), D. cultridens (Gervais \& Ameghino, 1880) y D. ensenadensis (Ameghino, 1888). La primera es una forma de gran tamaño con dientes carniceros proporcionalmente grandes (Caviglia, 1986; Berman \& Tonni, 1987; Trejo \& Jackson, 1998), que es claramente distinguible de D. gymnocercus. D. cultridens 
tiene un tamaño intermedio entre $D$. griseus y $D$. gymnocercus, presentando la rama horizontal de la mandíbula grácil y el $\mathrm{m} 1$ proporcionalmente grande (Berman, 1994: 168-176). D. ensenadensis sólo es conocida a partir del holotipo (ambas hemimandíbulas con la dentición casi completa) que se caracteriza principalmente por presentar una serie dentaria cerrada (sin diastemas) y dientes proporcionalmente grandes (Ameghino, 1888; Berman, 1994:182-186). Al tratarse de restos mandibulares no es posible contrastar la identidad del CICYTTP-PV-M-22 con D. cultridens y D. ensenadensis. Sin embargo, los caracteres utilizados para distinguir a las dos últimas especies fósiles presentan una variación considerable en las especies vivientes (obs. pers.) Claramente hace falta una nueva revisión de las especies fósiles, partiendo del estudio de la variabilidad intraespecífica de las especies vivientes de escaso porte. En este sentido los métodos estadísticos multivariados podrían ser una valiosa herramienta para explorar la variación morfométrica de las especies vivientes y en la comparación con ejemplares fósiles.

El estudio del ejemplar fósil permitió asignarlo a la especie viviente $D$. gymnocercus y constituye el primer registro de un cánido para el Lujanense de Entre Ríos y la Mesopotamia Argentina. Además, amplía la distribución geográfica de D. gymnocercus durante el Lujanense, llenando un vacío en su distribución durante esa edad.

\section{AGRADECIMIENTOS}

Agradecemos a R. Kemer por la donación del ejemplar estudiado; F.G. Aceñolaza (Programa de Biodiversidad del LitoralSECYT) y PICT 11928 (ANPCYT) por el apoyo financiero. Un árbitro anónimo y R. Fariña brindaron oportunas correcciones que contribuyeron a mejorar este trabajo.

\section{BIBLIOGRAFÍA}

Ameghino, F. 1888. Rápidas diagnosis de algunos mamíferos fósiles nuevos de la República Argentina. E.P. Coni, Buenos Aires, 17 pp.

Bechstein, J.M. 1799. Gemeinnützige Naturgeschichte Deutschlands nach allen drey Reichen. I. Säugethiere, Leipzig, 600 pp.

Berman, W.D. 1994. Los carnívoros continentales (Mammalia, Carnivora) del Cenozoico en la Provincia de Buenos Aires. Tesis Doctoral de la Facultad de Ciencias Naturales y Museo, Universidad Nacional de La Plata, 412 pp. (inédita).

Berman, W.D. \& Tonni, E.P. 1987. Canis (Dusicyon) avus Burmeister, 1864 (Carnivora, Canidae) en el Pleistoceno tardío y Holoceno de la Provincia de Buenos Aires. Aspectos sistemáticos y bioestratigráficos relacionados. Ameghiniana, 24, 245-250.

Berta, A. 1987. Origin, diversification, and zoogeography of the South American Canidae. Fieldiana: Zoology, 39 , 455-471.

Bowdich, T.E., 1821. An analysis of the natural classifications of Mammalia for the use of students and travelers. J. Smith, Paris, 115 pp.

Cabrera, A. 1931. On some South American canine genera. Journal of Mammalogy, 12, 54-67.

Cabrera, A. 1957. Catálogo de los Mamíferos de América del Sur. Parte I. Revista del Museo Argentino de Ciencias Naturales “Bernardino Rivadavia”, Zoología, 4, 1-307.

Caviglia, S. E. 1986. Nuevos restos de cánidos tempranos en sitios arqueológicos de Fuego-Patagonia. Anales del Instituto de la Patagonia, Serie Ciencias Sociales, 16, 85-93.

Cione, A. L. \& Tonni, E. P. 1999. Bioestratigraphy and chronological scale of uppermost Cenozoic in the Pampean Area, Argentina. In: Quaternary of South America and Antarctic Península (Eds. J. Rabassa \& M. Salemme). Quaternary vertebrate paleontology in South America, 12, 23-51.

Cione, A.L. \& Tonni, E.P. 2001. Correlation of Pliocene to Holocene southern South America and European vertebrate-bearing units. Bolletino della Società Paleontologica Italiana, 40, 167-173.

Clutton-Brock, J., Corbet, G.B. \& Hills, M. 1976. A review of the Family Canidae, with a classification by numerical methods. Bulletin of the British Museum (Natural History), Zoology, 29, 119-199.

Crespo, J.A. 1971. Ecología del zorro gris Dusicyon gymnocercus antiquus (Ameghino) en la provincial de La Pampa. Revista del Museo Argentino de Ciencias Naturales “Bernardino Rivadavia”, Ecología, 1, 147-205.

Fischer, G. 1814. Zoognosia tabulis synopticis illustrata. Volumen tertium. Quadrupedum reliquorum. Cetorum et montrymatum descriptionem continens. Nicolai Sergeidis Vsevolozky, Mosquae, 732 pp.

Fischer, G. 1817. Adversaria Zoologica. Mémoires de la Société impériale des naturalistes de Moscou, 5, 368-428.

Ginsberg, J. \& Macdonald, D. 1990. Foxes, Wolves, Jackals, and Dogs. An Action Plan for the Conservation of Canids. IUCN/SSC Canid Specialist Group - IUCN/SSC Wolf Specialist Group, Cambridge, 320 pp.

Hamilton Smith, C. 1839. Dogs. Canidae or genus Canis of authors. In: Jardine's Naturalist's Library, vol 9. W.H. Lizars impresor, Edinburgh, 267 pp.

Iriondo, M. 1996. Estratigrafía del Cuaternario de la Cuenca del Río Uruguay. In: Actas del XIII Congreso Geológico Argentino y III Congreso de Exploración de Hidrocarburos, 4, 15-25.

Iriondo, M., C. Ceruti \& R. Tardivo, 1985. Geomorfología y cuaternario del tramo inferior del Arroyo Feliciano. Revista de la Asociación de Ciencias Naturales de Litoral, Santa Fe, 16, 149-156.

Jayat, J.P., R.B. Barquez, M.M. Díaz \& P.J. Martínez. 1999. Aportes al Conocimiento de la Distribución de los Carnívoros del Noroeste de Argentina. Mastozoología Neotropical, 6, 15-30.

Kraglievich, L. 1930. Craneometría y clasificación de los 
cánidos sudamericanos, especialmente los argentinos actuales y fósiles. Physis, 10, 35-73.

Langguth, A. 1975. Ecology and evolution in the South American canids. In: The wild canids, their systematics, behavioral ecology and evolution (ed. M.N. Fox). Van Nostrand Reinhold, New York, 192-206.

Macdonald, D.W. \& Sillero-Zubiri, C. 2004. Dramatis personae. In: Biology and Conservation of Wild Canids (eds. D. W. Macdonald \& C. Sillero-Zubiri). Oxford University Press, Oxford, 3-36.

Noriega, J.I., Carlini, A.A. \& Tonni, E.P. 2004. Vertebrados del Pleistoceno tardío de la cuenca del Arroyo Ensenada (Departamento Diamante, provincia de Entre Ríos). In: Temas de la Biodiversidad del Litoral Fluvial Argentino (Ed. F.G. Aceñolaza). Instituto Superior de Correlación Geológica, Miscelánea, 12, 71-76.

Osgood, W.H. 1934. The genera and subgenera of South American canids. Journal of Mammalogy, 15, 45-50.

StatSoft, Inc., 2001. STATISTICA 6.0 (data analysis software system). www.statsoft.com.

Tedford, R., Taylor, B. E. \& Wang, X. 1995. Phylogeny of the Caninae (Carnivore: Canidae): the living taxa. American Museum Novitates, 3146, 1-37.

Trejo, V. \& Jackson, D. 1998. Cánidos patagónicos: identificación taxonómica de mandíbulas y molares del sitio arqueológico Cueva Baño Nuevo 1. Anales del Instituto de la Patagonia, Serie Ciencias Humanas, 26, 181-194.

Ubilla, M. \& Perea, D. 1999. Quaternary vertebrates of
Uruguay: A bioestratigraphic, biogegraphic and climatic overview. In: Quaternary vertebrate paleontology in South America (Eds. J. Rabassa \& M. Salemme). Quaternary of South America and Antarctic Península, 12, 75-90.

Van Gelder, R.G. 1978. A review of canid classification. American Museum Novitates, 2646, 1-10.

Wang, X., Tedford, R., Van Valkenburgh, B. \& Wayne, R.K. 2004. Ancestry. In: Biology and Conservation of Wild Canids (eds. Macdonald D.W. \& C. Sillero-Zubiri ). Oxford University Press, Oxford, 39-54.

Wayne, R. K., E. Geffen, D. J. Girman, K. P. Koepfli, L. M. Lau \& C. R. Marshall. 1997. Molecular systematics of the Canidae. Systematic Biology, 46, 622-653.

Winge, H., 1895. Jordfunde og nulevende rovdyr (Carnivora) fra Lagoa Santa, Minas Geraes, Brasilien. E Museo Lundii, 2 (4), 1-103.

Zrzavý, J. \& Řičánková, V. 2004. Phylogeny of recent Canidae (Mammalia, Carnivora): relative reliability and the utility of morphological and molecular datasets. Zoologica Scripta, 33, 311-333.

Zunino, G.E., Vaccaro, O.B., Canevari, M. \& Gardner, A.L. 1995. Taxonomy of the genus Lycalopex (Carnivora: Canidae) in Argentina. Proceeding of the Biological society of Wasshington, 108, 729-747.

Manuscrito recibido: 10 de Enero, 2005 Manuscrito aceptado: 17 de Septiembre, 2005

\section{APÉNDICE}

Especímenes y datos utilizados en los análisis multivariados; para las abreviaturas ver Tabla 1.

\begin{tabular}{|c|c|c|c|c|c|c|c|c|c|c|c|c|c|c|c|c|c|c|}
\hline & & LCB & LCR & LP & LR & LB & AIO & $\mathrm{CPO}$ & APPO & AR & AM & $\mathrm{ACO}$ & SDS & LC & LP4 & AP4 & LM1 & AMI \\
\hline MACN 20207 & D. griseus & 125,4 & 129,5 & 67,53 & 54,56 & 19,52 & 22,71 & 24,48 & & 17,53 & 36,58 & 24,26 & 58,69 & 5,66 & 11,06 & 4,62 & 8,1 & 11,7 \\
\hline MACN 20206 & D. griseus & 116,8 & 120,4 & 60,97 & 51,18 & 19,4 & 20,33 & 23,44 & 27,65 & 17,65 & 33,57 & 21,8 & 54,67 & 5,83 & 10,46 & 4,96 & 6,35 & 11,41 \\
\hline MACN 20277 & D. griseus & 125,8 & 129,1 & 66,26 & 54,97 & 19,1 & 20,34 & 23,97 & 29,13 & 18,42 & 36,05 & & 58,53 & 6,25 & 11,27 & 6,22 & 7,97 & 11,9 \\
\hline MACN 20208 & D. griseus & 122,2 & 126,8 & 64,74 & 53,14 & 19,18 & 25,03 & 26,87 & 33,52 & 18,59 & 34,7 & 23,8 & 57,32 & 5,3 & 11,07 & 5,02 & 6,81 & 11,65 \\
\hline $\mathrm{MACN} 23.19$ & D. griseus & 116 & 120,3 & 61,66 & 49,45 & 18,08 & 21,59 & 23,7 & 30,21 & 17,6 & 34,8 & 21,9 & 55,49 & 4,8 & 10,83 & 5,43 & 7,19 & 12,04 \\
\hline MACN 20278 & $D \cdot$ griseus & 120,9 & 125,7 & 63,45 & 50,88 & 20,09 & 21,29 & 25,5 & 30,54 & 18,55 & 35,26 & 24,25 & 55,31 & 5,19 & 10,85 & 5,3 & 7,66 & 11,13 \\
\hline MACN 7.41 & $D$. griseus & 113,4 & 116,5 & 60,81 & 48,57 & 20,04 & 19,95 & 23,72 & 28,32 & 17,24 & 32,95 & 22,66 & 51,31 & 4,33 & 10,93 & 5,04 & 6,57 & 10,93 \\
\hline MACN 2479 & D. griseus & 126,9 & 132,1 & 68,76 & 55,14 & 19,63 & 20,82 & 21,24 & 27,97 & 19,78 & 35,59 & 23,52 & 59,97 & 5,25 & 12,42 & 5,39 & 8,88 & 12,02 \\
\hline MACN 226 & D. griseus & 117,9 & 120,5 & 63,01 & 50,82 & 18,72 & 20,82 & 23,83 & 29,7 & 17,97 & 35,18 & 23,26 & 56,33 & 4,69 & 10,58 & 5,05 & 7,01 & 11,47 \\
\hline MACN 24.66 & D. griseus & 124,8 & 127,4 & 64,68 & 54,95 & 20,21 & 20,9 & 23,68 & 29,89 & 18,48 & 36,19 & 23,57 & 58,45 & 5,21 & 12,3 & 5,78 & 7,83 & 11,9 \\
\hline MACN 223 & D. griseus & 121,7 & 125,2 & 64,65 & 54,45 & 18,89 & 20,55 & 24,09 & 27,76 & 17,13 & 34,79 & 21,51 & 57,1 & 5,18 & 10,99 & 4,72 & 8,07 & 12,2 \\
\hline MACN 15692 & D. griseus & 131,8 & 138,4 & 72,84 & 59,23 & 19,04 & 24,23 & 25,1 & 30,17 & 19,79 & 38,16 & 25,21 & 60,81 & 5,86 & 11,8 & 5,55 & 7,08 & 12,35 \\
\hline MACN 14902 & D. griseus & 116,9 & 120,4 & 61,4 & 49 & 18,69 & 21,45 & 24,6 & 32,38 & 18,8 & 34,3 & 23,09 & 54,85 & 4,94 & 11,67 & 5,22 & 7,29 & 11,58 \\
\hline MACN 24.51 & D. griseus & 122,4 & 126,4 & 66,41 & 54,16 & 18,97 & 20,62 & 24,54 & 28,67 & 17,7 & 34,27 & 21,76 & 56,53 & 5,4 & 10,66 & 5,31 & 7,23 & 11,42 \\
\hline MACN 20276 & D. griseus & 127 & 132,4 & 65,57 & 55,66 & 21,26 & 23,15 & 24,92 & 33,05 & 18,59 & 36,66 & 23,16 & 58,22 & 5,77 & 11,74 & 4,99 & 8,73 & 12,12 \\
\hline MACN 16321 & D. griseus & 121 & 125,7 & 64,74 & 52,14 & 19,85 & 23,25 & 25,48 & 29,2 & 20,67 & 37,69 & 24,03 & 57,54 & 6,07 & 12,29 & 5,87 & 7,87 & 11,78 \\
\hline MACN 20205 & D. griseus & 124,3 & 126,7 & 67,85 & 53,71 & 18,76 & 25,29 & 22,89 & 29,56 & 18,13 & 34,79 & 22,94 & 58,55 & 5,37 & 11,88 & 5,92 & 9,2 & 12,46 \\
\hline MACN 24.81 & D. griseus & 121,3 & 127,8 & 67,71 & 53,44 & 19,83 & 21,38 & 24,12 & 33,09 & 19,2 & 34 & 24,39 & 57,57 & 5,31 & 11,19 & 4,66 & 6,96 & 10,46 \\
\hline MACN 7.40 & D. griseus & 114,7 & 118,1 & 61,43 & 47,78 & 18,58 & 19,98 & 23,15 & 28,45 & 16,5 & 33,69 & 23,61 & 54,39 & 6,1 & 11,75 & 5,92 & 9,26 & 12,71 \\
\hline MACN 20814 & D. griseus & 130 & 133,6 & 69,53 & 56,13 & 21,94 & 23,04 & & & 19,65 & 36,98 & 25,66 & 59,99 & 5,5 & 12,27 & 5,77 & 7,99 & 12,68 \\
\hline MACN 3.63 & D. griseus & 110 & 113,8 & 57,92 & 47,82 & 18,21 & 21,14 & 24,54 & 27,43 & 17,04 & 32,65 & 20,16 & 50,6 & 4,6 & 10,98 & 5,36 & 7,01 & 10,76 \\
\hline MLP S/N & D.griseus & 132,3 & 134,9 & 69,72 & 56,41 & 20,22 & 23,29 & 23,08 & 31,99 & 20,48 & 38,92 & 26,76 & 59,29 & 7,52 & 11,68 & 5,99 & 9,18 & 11,52 \\
\hline MLP S/N $N^{\circ}$ & D. griseus & 121 & 123,5 & 66,78 & 52,96 & 19,69 & 21,18 & 22,64 & 30,4 & 18,6 & 34,22 & 21,4 & 55,96 & 5,36 & 10,95 & 5,04 & 7,58 & 10,76 \\
\hline MLP S/N" & D. griseus & 120,5 & 125,7 & 64,13 & 50,48 & 18,04 & 19,99 & 21,56 & 25,35 & 19,43 & 37,15 & 24,47 & 57,14 & 6,64 & 11,12 & 5,74 & 7,99 & 11,16 \\
\hline MLP 4-VIII-98-4 & ${ }^{\prime} D$. griseus & 118 & 116,1 & 59,86 & 48 & 16,87 & 19,35 & 22 & 26,44 & 18,1 & 36,79 & 24,06 & 52,18 & 5,89 & 11,41 & 5,21 & 6,81 & 11,3 \\
\hline MLP S $/ \mathbb{N}^{\circ}$ & D. culpaeus & & 180,1 & 93,37 & 77,19 & & 30,82 & 27,23 & 37,05 & 30,65 & 51,02 & & 75,19 & 12,28 & 16,95 & 8,2 & 9,46 & 14,97 \\
\hline MLP S $/ \mathbb{N}^{\circ}$ & D. culpaeus & 162,3 & 173 & 86,26 & 71,98 & 21,3 & 25,65 & 23,21 & 31,46 & 26,44 & 43,76 & 27,15 & 68,79 & 11,36 & 15,52 & 7,54 & 8,23 & 13,51 \\
\hline $\operatorname{MLP} \mathbf{S} / \mathrm{N}^{\circ}$ & D. culpaeus & 158,3 & 164,7 & 84,38 & 69,89 & 21,53 & 26,22 & 27,4 & 35,04 & 27,17 & 47,31 & 30,17 & 72,09 & 9,04 & 15,95 & 6,48 & 8,38 & 14,21 \\
\hline MLP 1264 & D. culpaeus & 172,6 & 178,2 & 93,72 & 76,29 & 25,63 & 33,01 & 29,22 & 41,19 & 28,82 & 50,51 & 30,87 & 76,14 & 9,69 & 14,9 & 7,17 & 9,52 & 13,74 \\
\hline
\end{tabular}




\begin{tabular}{|c|c|}
\hline MLP 5-III-36-9 & D. culpaeus \\
\hline MLP 27-X-97-19 & D. culpaeus \\
\hline MLP 1749 & D. culpaeus \\
\hline MLP 27-X-97-10 & D. culpaeus \\
\hline MLP 1265 & D. culpaeus \\
\hline MLP 1266 & D. culpaeus \\
\hline MLP 1267 & D. culpaeus \\
\hline MLP S/N $\mathbb{N}^{\circ}$ & D. culpaeus \\
\hline MACN-Ma 15035 & D.culpaeus \\
\hline MACN-Ma 15044 & D. culpaeus \\
\hline MACN-Ma 15059 & D. culpaeus \\
\hline MACN-Ma 15056 & D. culpaeus \\
\hline MACN-Ma 15045 & D. culpaeus \\
\hline MACN-Ma 15024 & D. culpaeus \\
\hline MACN-Ma 15050 & D. culpaeus \\
\hline MACN-Ma 15049 & D. culpaeus \\
\hline MACN-Ma 15082 & D.culpaeus \\
\hline MACN-Ma 15092 & D. culpaeus \\
\hline MLP 15760 & D. gymnocercus \\
\hline MACN-Ma 15761 & D. gymnocercus \\
\hline MACN-Ma 15762 & D. gymnocercus \\
\hline MACN-Ma 15763 & D. gymnocercus \\
\hline MACN-Ma 15764 & D. gymnocercus \\
\hline MACN-Ma 15757 & D. gymnocercus \\
\hline MACN-Ma 15758 & D. gymnocercus \\
\hline MACN-Ma 15759 & D. gymnocercus \\
\hline MACN-Ma 15765 & D. gymnocercus \\
\hline MACN-Ma 15766 & D. gymnocercus \\
\hline MLP 8-X-92-15 & D. gymnocercus \\
\hline MLP 13-IV-99-28 & D. gymnocercus \\
\hline MLP I-III-95-1 & D. gymnocercus \\
\hline MLP 13-IV-99-18 & D. gymnocercus \\
\hline MLP 8-IV-99-3 & D. gymnocercus \\
\hline MLP 16-111-99-24 & D. gymnocercus \\
\hline MLP 26-V-95-4 & D. gymnocercus \\
\hline MLP 16-111-99-18 & D. gymnocercus \\
\hline MLP 16-III-99-14 & D. gymnocercus \\
\hline MLP 8-IV-99-8 & D. gymnocercus \\
\hline MACN-Ma 15927 & D. gymnocercus \\
\hline MACN-Ma 15928 & D. gymnocercus \\
\hline MACN-Ma 15929 & D. gymnocercus \\
\hline MACN-Ma 15938 & D. gymnocercus \\
\hline MACN-Ma 15939 & D. gymnocercus \\
\hline MACN-Ma 15923 & D. gymnocercus \\
\hline MACN-Ma 15924 & D. gymnocercus \\
\hline MACN-Ma 15925 & D. gymnocercus \\
\hline MACN-Ma 15926 & D. gymnocercus \\
\hline MACN-Ma 15930 & D. gymnocercus \\
\hline
\end{tabular}

\begin{tabular}{|c|c|c|c|c|c|c|c|c|c|c|c|c|c|c|c|c|}
\hline 60,9 & 69,3 & 83,7 & 70,63 & 24,03 & 27,76 & 29,3 & 37,77 & 26,99 & 47,37 & 29,78 & 72,36 & 10,4 & 15,73 & 7,05 & 8,9 & 14,34 \\
\hline 70,9 & 79,1 & 1,65 & 75,4 & 23,37 & 32,42 & 27,3 & 41,77 & 28,38 & 48,93 & 30,92 & 75,82 & 11,87 & 17,75 & 7,99 & & 13,95 \\
\hline 86,5 & 95,5 & 01,3 & 2,07 & 4,43 & 2,84 & 8,47 & 2,06 & 30,39 & 51,02 & 29,82 & 7,36 & 11,65 & 6,96 & 7,49 & & 446 \\
\hline 68,4 & 78,1 & 89,95 & 76,7 & 22,68 & 31,2 & 4,87 & 37,72 & 27,6 & 47,46 & 30,09 & 3,51 & 10,67 & 14,62 & 5,93 & 63 & 3,67 \\
\hline 55,4 & 64,3 & 83,29 & 67,92 & 21,6 & $8,8,38$ & 26,55 & 36,41 & 26,51 & 46,26 & 30,2 & 69,44 & 10,13 & 15,55 & 7,05 & 3,29 & 3,57 \\
\hline 174 & 79,9 & 93,35 & 79,32 & 23,94 & 29,36 & 26,88 & 38,73 & 29,27 & 48,91 & 30,63 & 78,89 & 10,8 & 16,67 & 7,11 & 26 & 14,3 \\
\hline 157,7 & 66,3 & 83,83 & 69,42 & 22,15 & 29,22 & 27,33 & 35,56 & 26,2 & 44,74 & 27,99 & 71,97 & 9,03 & 14,87 & 5,51 & 8,84 & 13,71 \\
\hline 164 & 74,5 & 86,93 & 72,85 & 23,83 & 1,93 & 26,43 & 38,55 & 30,17 & 49,3 & 31,38 & 71,38 & 10,6 & 16,68 & 7,17 & ,96 & 0,4 \\
\hline 68,1 & 77,6 & 89,54 & 74,94 & 23,9 & 28,9 & 25,85 & 41,03 & 29,12 & 48,96 & 31,07 & 76,6 & 9,55 & 15,69 & 7,84 &, 39 & 4,68 \\
\hline 68,5 & 79,5 & 90,14 & 76,97 & 22,31 & 32,17 & 31,14 & 40,73 & 29,75 & 49,71 & 29 & 76,05 & 9,36 & 15,16 & 7,28 & 3,99 & 14,8 \\
\hline 59,1 & 67,5 & 85,29 & 69,28 & 22,13 & 28,33 & 27,36 & 38,06 & 28,08 & 48,05 & 29,93 & 68,31 & 8,39 & 15,76 & 7,26 & 05 & 4,33 \\
\hline 67,2 & 175 & 87,51 & 74,06 & 23,86 & 27,41 & 26,88 & 35,88 & 28,51 & 48,09 & 29,25 & & 9,98 & & 8,74 &, 18 & 4,15 \\
\hline 65,4 & 172,9 & 84,69 & 72,8 & 24,06 & 27,03 & 27,31 & 34,83 & 26,64 & 46,13 & 29,38 & 73,84 & 8,91 & 14,23 & 6,93 &, 88 & 13,4 \\
\hline 62,7 & 69,7 & 84,29 & 71,95 & 21,81 & 7,65 & 26,1 & & 27,43 & & 29,83 & & 9,18 & & 6,87 & ,48 & 3,07 \\
\hline 61,2 & 170,8 & 84,79 & 72,25 & 22,69 & 28,74 & 26,13 & 35,61 & 27,26 & & 29,98 & & 8,85 & 5,34 & 6,5 & ,58 & 13,36 \\
\hline 54,1 & 163 & 78,23 & 66,8 & 21,71 & 28,55 & 24,95 & 37,08 & 25,41 & 41 & 29,63 & 68,05 & 8,96 & 13,77 & 6,48 &, 19 & 12,43 \\
\hline 77,9 & 187,3 & 92,98 & 79,83 & 23,19 & 29,15 & 26,8 & 34,74 & 28,12 & & 30,24 & & 9,19 & & 7,31 & 3,65 & 13,01 \\
\hline 72,2 & 81,1 & 89,9 & 75,59 & 22,79 & 29,71 & 25,33 & 36,53 & 28,46 & 46,81 & 30,34 & 74,75 & 10,14 & 14,73 & 7 & 8,53 & 13,78 \\
\hline 31,4 & 36,9 & 68,3 & 57,1 & 20,4 & 22,8 & 23,3 & 33,6 & 20,7 & & 25 & 7,3 & & & 5,1 & 6,9 & 10,2 \\
\hline 32,7 & 36,6 & 69,6 & 57,7 & 21,1 & 24,6 & 24,5 & 34 & 21,2 & & & & & & 5 & & 0,7 \\
\hline 134,1 & 38,1 & 70,3 & 57,6 & 21,5 & 25,9 & 27,3 & 36,3 & 22,3 & & & 0,5 & & 14 & & & 11,2 \\
\hline 31,6 & 35,4 & 70,4 & 57,1 & 19,4 & 23,2 & 25,4 & 33,7 & 21 & & & & 5 , & & & J, & 10,9 \\
\hline 136,5 & 38,9 & 71,7 & 59,2 & 21,2 & 27 & 22,7 & 38,3 & 22,8 & & 26,1 & 60,7 & 6,9 & & 5,9 & $\lambda, 3$ & 11,6 \\
\hline 148,2 & 53,7 & 76,2 & 64,8 & 23,4 & 25,5 & 23,9 & 35,2 & 23,4 & 38,5 & 27,5 & 64,1 & 7 & 13,2 & 5,7 & 8,4 & 12,2 \\
\hline 133,6 & 139,3 & 70,5 & 56,6 & 21,8 & 23,1 & 21,6 & 33 , & 22,8 & 35, & 27 & 59,2 & 6,6 & 11,9 & 5 & 7,9 & 12 \\
\hline 130,6 & 137,4 & 68,5 & 57,6 & 19,9 & 27,5 & 27,1 & 38 & 21,2 & 37,5 & 24,4 & 59 & & 1,9 & 5,8 & 7,3 & 11,2 \\
\hline 138,7 & 42,1 & 73,2 & 59,3 & 22,1 & 24,2 & 24,3 & 33 & 22,8 & 38,4 & 25,1 & 63,7 & 6,7 & 13,6 & 6 & 7,7 & 12,3 \\
\hline 132,1 & 35,2 & 68 & 55,2 & 22,2 & & 19,4 & 28 & 20,7 & 39 & 25,6 & 57 & 6,2 & & 5,3 & 7,9 & 11,4 \\
\hline 142,6 & 50,2 & 75,4 & 62,8 & 21,3 & 26,9 & 24,9 & 40,6 & 24,2 & & 32,9 & & 7 & & 6,3 & 8 & 12,2 \\
\hline 127,8 & 33,2 & 66,2 & 55,6 & 20 & 25,7 & 24,4 & 32 & 21,1 & 37,2 & 29,6 & 56,7 & 5,5 & 11,9 & 5,6 & 7,6 & 12 \\
\hline 138,6 & 142,3 & 71,5 & 61,1 & & 27,4 & 27,7 & 3 & 22,4 & 39 & 32,8 & 60,6 & 6 , & 12,8 & 6,3 & 8,5 & 12,6 \\
\hline 139,2 & 144 & 71,6 & 60,9 & 21,4 & 25,5 & 23 & 35,3 & 21,4 & 38,2 & 29,6 & 61 & 6,8 & 12,1 & 6,4 & 7,9 & 12,2 \\
\hline 138,1 & 143,5 & 73,6 & 60,4 & 22,4 & 26,7 & 25,8 & & 23,6 & 39,7 & 33 & 61,6 & 6,4 & 2,4 & 6,1 & 8,9 & 12 \\
\hline 148 & 155 & 77,8 & 67 & 23,4 & 28,2 & 25,5 & & 24,2 & & 34,1 & 66,4 & 7,5 & & 6,7 & 9,4 & \\
\hline 141,2 & 144,2 & 73,7 & 60,1 & 21,3 & & 24 & 36,7 & 21,9 & 40,1 & 31,1 & & & & 7 & 8,9 & 13,4 \\
\hline 143,9 & 150,1 & 76 & 62,6 & 21,6 & 27,7 & 25,6 & 37,7 & 23,8 & 41,2 & 32,9 & 63,5 & 6,8 & 12,5 & 5,8 & 8,7 & 13 \\
\hline 148,6 & 157 & 77,1 & 64,8 & 21,7 & 27,6 & 24,4 & & 23,1 & & 32,8 & 65,5 & 7 & 12 & 5,9 & 9,3 & 12,7 \\
\hline 148,1 & 153,1 & 76,4 & 66,5 & 22,5 & 25,9 & 22,8 & 35,2 & 25,6 & 42,6 & 30 & 66,1 & 8,1 & 12,5 & 7 & 8,8 & 13,1 \\
\hline 128,1 & 131,6 & 65,4 & 56,2 & 20,4 & 24,2 & 24,3 & & 19,8 & & 24,5 & 57,6 & & & 5,7 & 7,7 & 10,7 \\
\hline 125,6 & 129,8 & & 53,5 & & & 27 & & & & 25,9 & 56,3 & 5 & 11,2 & 5,3 & 8,2 & 12 \\
\hline 138,7 & 143,8 & 72,3 & 61,8 & 22,8 & & 20,5 & 31 , & 20,9 & 37,5 & 26,6 & 62,6 & 6,1 & & 6,3 & 7,6 & 11,3 \\
\hline 124,9 & 131,4 & 64,8 & 54,7 & 19,1 & 22,4 & 22,8 & & & & 24,6 & & & & 5,9 & 7,9 & 13,2 \\
\hline 134,9 & 138,8 & 70 & 58,5 & 20,1 & 25,6 & 26,5 & 33,8 & 22,4 & 38,8 & 26,3 & 61,2 & 6,2 & 11,9 & 5,5 & 8,2 & 12,9 \\
\hline 130,6 & 136,4 & 66,4 & 56,4 & 22,7 & 26,7 & 26 & 38,1 & 21,2 & 38,8 & 25,7 & 59 & 5,8 & 11,2 & 5,2 & 7,4 & 11,1 \\
\hline 137,8 & 141,7 & 69,8 & 58,2 & 22,2 & & 26,8 & 38 & 24 & 41 & 26,7 & 60,8 & 6,6 & 12,3 & 6,7 & 8,6 & 12,9 \\
\hline 141,5 & 146 & 74,5 & 62,4 & 21,4 & 26,1 & 26,7 & 38,9 & & 39,7 & 25,3 & 63,6 & 6,4 & 12,9 & 6 & 8,2 & 13 \\
\hline 143,7 & 147,2 & 73,2 & 61,6 & 21,3 & 26,5 & 24,3 & 34,6 & 21,7 & 39,7 & 26 & 61,7 & 6,4 & 12,7 & 5,8 & 9,1 & 12,8 \\
\hline 143,2 & 146,4 & 73,8 & 60,7 & 21,4 & 27,6 & 27,2 & 36,6 & 24,3 & 39,8 & 25,7 & 62 & 7 & 13,3 & 6,5 & 8,3 & 12,7 \\
\hline
\end{tabular}

